systolic, and 90-100 diastolic. The spinal fluid was negative. $\mathrm{X}$-ray verified the presence of an abdominal tumour, and operation revealed a malignant hypernephroma at upper pole of right kidney. The patient died. Autopsy showed a very small fibroid thymus. The lungs showed secondary deposits. Section of the tumour revealed a complete absence of normal suprarenal tissue. Some areas resembled a suprarenal cortex, others a medullary carcinoma; others still resembled an angiosarcoma. The ovaries showed, on section, an unusual number of large cystic follicles, but no evidence of menstruation. The brain on examination showed small areas on the surface which appeared softened. The basilar artery showed a small patch of thickening of its wall, and on section some splitting of the elastic layers. The intima was irregularly thickened with a glistening refractive substance, which Herxheimer fat stains showed to contain heavy deposits of lipoids. This patchy intimal thickening partly occluded the vessel lumen. The pia was thickened and cellular, the veins congested and their walls thickened. All the vessels in the cortex showed a marked sclerosis. There were small areas of softening. After discussing possible relations between brain development and the adrenals, and pointing out the association of tuberose sclerosis with hypernephroma and adenomata of the adrenals and of Alzheimer's disease with hypothyroid states, the author wonders, had this case lived, would it have developed into one of Alzheimer's disease?

G. W. T. H. Fleming.

\title{
The Anatomo-Pathological Basis of the Parkinsonian Syndrome following Epidemic Encephalitis. (Brain, December, 1926.) McAlpine, $D$.
}

In a preliminary summary of the published accounts of the pathology of the Parkinsonian syndrome, the author points out that the consensus of opinion favours the substantia nigra as the chief area affected.

He then gives a summary of present knowledge of the anatony of the substantia nigra, quoting Foix and Nicolesco. The pigment does not make its appearance until after the first few years of life, and is not abundant until after 20. There are two parts, the zona reticulata, consisting mainly of cell processes, and the zona compacta, which comprises the main body of the substantia nigra. The cells are large and are grouped in three islets-external, middle and internal. The cells of the inner group are small compared with the other two. The pigment is generally grouped into a mass which lies towards one of the poles of the cell. It is from this pole that the axone arises. The efferent fibres fall into two groups. The first comprises those fibres that penetrate into the midst of the pyramidal fibres, and then turn downwards, linking up with the centres lower in the mid-brain. The second group contains those fibres that form the peduncle of the substantia nigra. Some of these fibres mingle with those of the posterior commissure, and serve as commissural fibres between each substantia nigra. They 
may also connect the substantia nigra with other nuclei on the opposite side of the mid-brain. Foix and Nicolesco think that some fibres of the peduncle do not cross in the posterior commissure, but descend and come into relationship with grey masses lower down in the brain-stem. In regard to afferent fibres, descending fibres come from the corpus striatum, particularly the globus pallidus. There may be fibres from the cortex, but these are unimportant. In his series of eight cases of encephalitis lethargica the author found that the cortex, thalamus and corpus striatum were practically normal. The substantia innominata of Reichert, the corpus Luysii and upper part of the red nucleus were normal, or only showed a slight increase in glial content. In the midbrain, the area occupied by the substantia nigra was poorly delimited and appeared paler. In some cases the cells had almost disappeared; those that remained showed chromatolysis. In other cases groups of cells were to be found, some of which were normal. In some cases quantities of pigment were lying free in the parenchyma. There was, except in one case, an increase in the number of glial cells. The gliosis was practically confined to the substantia nigra and the peri-aqueductal grey matter in the pons and mid-brain. Neuroglial fibres were especially plentiful in the region of vessels. Marked cuffing of the vessels only occurred in two out of the eight cases. The capillaries were present in excessive numbers. The nerve-fibres in Weigert-Pal sections showed alterations in their myelin sheaths. In some cases there was a thinning out of the fibre network; in others the fibres were interrupted in their course, the myelin sheath appearing as a series of beads. The pons showed a gliosis in the grey matter underlying the aqueduct of Sylvius; this process spread out laterally and of ten involved the locus caruleus. In two cases the cells of the locus caruleus were almost entirely destroyed on one side. In some cases there was depigmentation with chromatolysis of some of the cells of the locus caruleus. The author thinks the changes in the locus caruleus may be responsible for some of the symptoms attributed to the vegetative system.

G. W. T. H. Fleming.

\section{Mental Deficiency.}

The Nature of Hereditary Mental Defect. (Proc. Amer. Assoc. for the Study of the Feeble-minded, 1926.) Davenport, Charles B.

Concerning the exact nature of inheritance in the feeble-minded we are, says Dr. Davenport, still very ignorant. "If," he writes, "we adopt the hypothesis that prehistoric man had not gained all of the mental capacities that have appeared in some of his descendants, it is possible that he has left other descendants that remain more nearly on his intellectual plane."

As to the specific question whether mental defect is inherited in Mendelian fashion, Dr. Davenport is of the opinion that the subject deserves further study. The need, he maintains, is for more methods of measuring mentality. When these have been evolved 